\title{
Differential susceptibility of Type 1 and Type 4 gonococci to chlorhexidine (Hibitane)
}

\author{
SHEENA A. WAITKINS* AND I. GEARY \\ Department of Medical Microbiology, University of Sheffield Medical School
}

In recent years several reports have appeared in the literature advocating the use of chemical prophylactic measures to control the current epidemic of gonorrhoea (Lee, Utidjian, Singh, Carpenter, and Cutler, 1972; Cowan and Cree, 1973; Lee, 1974). As yet, no ideal agent has been found; we present results in this paper which suggest that Hibitane could be used as an effective prophylactic agent.

Hibitane (chlorhexidine) is a 1, 6-di C4-chlorophenyldiguanido hexane. Its salts are distinguished by their wide range of antimicrobial activity, being highly effective against both Gram-positive and Gram-negative organisms. As with other germicides, chlorhexidine is reduced somewhat in antimicrobial activity by certain organic substances notably milk, blood, and pus; however, this reduction is less marked than that observed with some of the commonly used germicides (Calman and Murray, 1956).

Chlorhexidine, when used in effective germicidal concentrations, has been reported to have very little, if any, toxic effect on animal tissues (I.C.I. Pharmaceuticals Division, 1973). Various forms of Hibitane have been used as skin disinfectant (Murray and Calman, 1955; Lowbury, Lilly, and Bull, 1960). Studies of Hibitane as a cleansing agent in wounds, burns, and skin infections have also been reported (Montgomery, 1964; Fowler, 1963; Grant, 1968), and its usefulness has been established in obstetrics and gynaecology. Calman and Murray (1956) compared Hibitane with a number of other antiseptics commonly used in midwifery and found it to have a very much safer margin. Barr (1964) stated that in general practice obstetrics swabbing the genitourinary area with Hibitane was non-irritant and effective. Morris and McLaren (1969), in a comparative trial against commonly used deodorants, found that chlorhexidine vaginal sprays were superior

Received for publication October 7, 1974

^Present address: Neisseria Department, Statens Serum Institute, Copenhagen

Address for reprints: Mr. I. Geary, Department of Medical Microbiology, University of Sheffield Medical School, Beech Hill Road, Sheffield, S10 2RX because they eliminated Gram-negative organisms which produced the odours. No spermicidal activity seems to have been reported with Hibitane used as a genital spray.

This paper presents a study of Hibitane, in the form of chlorhexidine gluconate solution, as a potential prophylactic chemical in the control of gonorrhoea. However, it soon became apparent to us that Hibitane exerted a differential bactericidal action on virulent and non-virulent gonococci; virulent strains of Type 1 were more susceptible to the killing action of Hibitane than strains of Type 4. This could prove of value as a differential marker between the gonococcal types.

\section{Material and methods}

MEDIA USED

5 per cent. lysed horse blood agar

Columbia agar base (Oxoid) 39 g., distilled water $1,000 \mathrm{ml}$. Soak for $15 \mathrm{~min}$. Mix and autoclave at $121^{\circ} \mathrm{C}$. for $15 \mathrm{~min}$. Allow to cool to $55^{\circ} \mathrm{C}$. and then add $50 \mathrm{ml}$. sterile horse blood (Oxoid) lysed by repeated freezing and thawing.

Difco G.C. medium base plus defined supplement (GCMB medium)

Difco G.C. medium base $36 \mathrm{~g}$., distilled water $1,000 \mathrm{ml}$. Heat to $70^{\circ} \mathrm{C}$. for $10 \mathrm{~min}$. Autoclave at $121^{\circ} \mathrm{C}$. for $15 \mathrm{~min}$. Allow to cool to $60^{\circ} \mathrm{C}$. and add $20 \mathrm{ml}$. supplement (White and Kellogg, 1965).

\section{'Lab Lemco' broth}

Lab Lemco broth granules (Oxoid) 8 g., distilled water $1,000 \mathrm{ml}$. Mix well and distribute in $5 \mathrm{ml}$. amounts. Autoclave at $121^{\circ} \mathrm{C}$. for $15 \mathrm{~min}$.

\section{Serum broth}

Lab Lemco broth prepared as above plus 10 per cent. inactivated horse serum adjusted to $\mathrm{pH} 4.5$.

Phosphate buffered saline (PBS) pH 7.3

Dulbecco saline was prepared (Dulbecco and Vogt, 1954). Dulbecco tablets A and B Oxoid were made up as described by the manufacturers and sterilized by autoclaving. 


\section{Hibitane solution}

Hibitane gluconate 20 per cent. solution (chlorhexidine gluconate solution B.P.) obtained from I.C.I. This was sterilized by autoclaving at $115^{\circ} \mathrm{C}$. for $30 \mathrm{~min}$. Final concentrations were made up in Dulbecco solution as shown in Table $I$.

\section{NEUTRALIZING AGENT}

Lecithin $3 \mathrm{~g}$., alcohol $3 \mathrm{ml}$. (methanol), Tween-80 $1 \mathrm{ml}$., Lab Lemco (prepared as above) $96 \mathrm{ml}$. The lecithin was dissolved in alcohol by heating in a water bath and the broth then added. Heating was continued until mixing was complete. Tween-80 was then added and the solution was sterilized by autoclaving at $115^{\circ} \mathrm{C}$. for $15 \mathrm{~min}$. Just before use the neutralizing agent was diluted 1:2 with sterile distilled water.

TEST ORGANISMS

All strains of Neisseria gonorrhoeae were isolated from patients attending the Sheffield V.D. Clinic and cultured initially on 5 per cent. lysed horse blood agar with vancomycin ( $3 \mu \mathrm{g} . / \mathrm{ml}$.). They were identified by Gram's stain, a positive oxidase reaction, and the ability to ferment glucose but not maltose or sucrose. Fermentation tests were performed using a modified carbohydrate medium containing Difco GC medium base with 1 per cent. sugar concentrations (Flynn and Waitkins, 1972). After one subculture had shown the strains to be pure they were then selected for colonial Types 1 and 4 (Kellogg, Peacock, Deacon, Brown, and Pirkle, 1963).

PREPARATION OF ORGANISMS FOR EXPERIMENTS

$N$. gonorrhoeae were grown in Difco G.C. medium base for $18 \mathrm{hrs}$ at $37^{\circ} \mathrm{C}$. in a 5 per cent. $\mathrm{CO}_{2}$ atmosphere. Colony types were checked using a Zeiss-stereoscan IV and the organisms were then scraped off and washed three times in warmed Dulbecco phosphate saline pH 7:3. The deposit was re-suspended in $10 \mathrm{ml}$. Dulbecco saline and the turbidity of suspensions measured on an E.E.L. nephelometer. The bacterial counts were estimated by reference to previously prepared graphs, to give a standard curve of approximately $1 \times 10^{4}$ organisms $/ \mathrm{ml}$. These counts were confirmed by the Miles and Misra method on 5 per cent. lysed horse blood agar (Miles, Misra, and Irwin, 1938).

\section{EXPERIMENTAL PROCEDURE}

\section{Hibitane solution}

Dilutions of Hibitane gluconate in PBS were made from $1 / 1,000$ to $1 / 10,000$.

\section{Experiment}

The experimental procedure was that shown diagramatically in the Figure. To each dilution of Hibitane solution was added $0.5 \mathrm{ml}$. bacterial suspension. The mixture was allowed to react for $2 \frac{1}{2} \mathrm{~min}$. before being stopped by the
$1 \quad 0.5 \mathrm{ml}$ bacterial suspension odded to $4.5 \mathrm{ml}$ of each Hibitane dilution

2

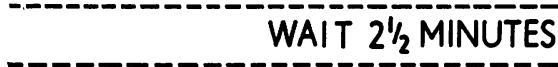

-

3 Add $5 \mathrm{ml}$ neutralizer

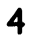

5

6
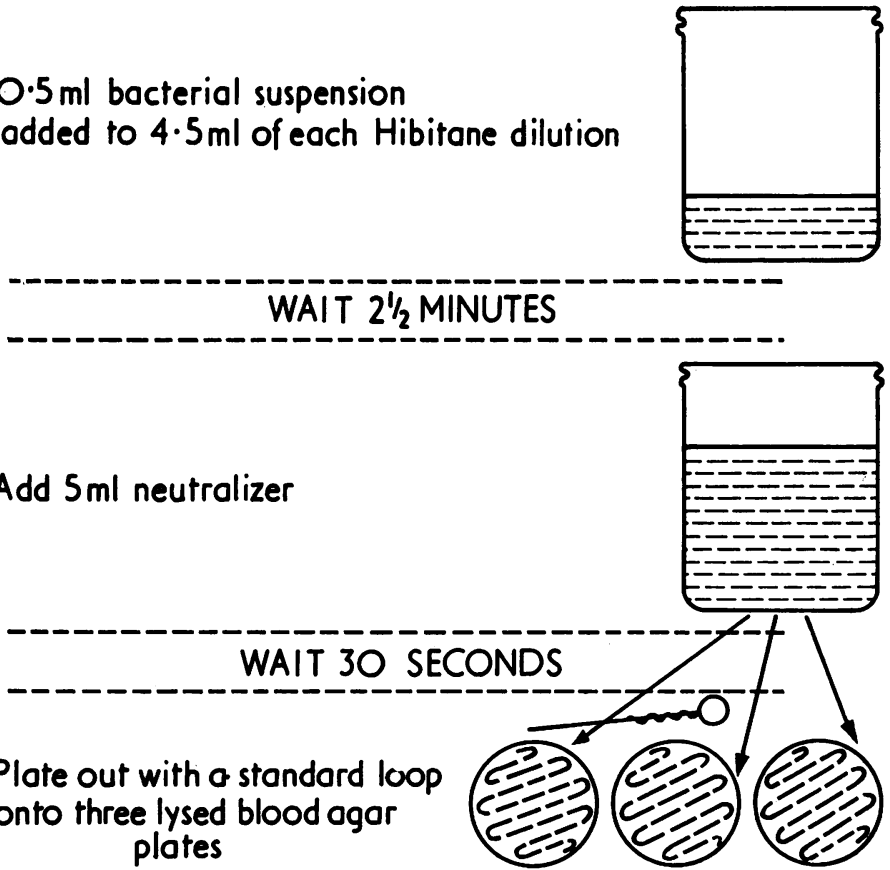

INCUBATE FOR 18 HRS

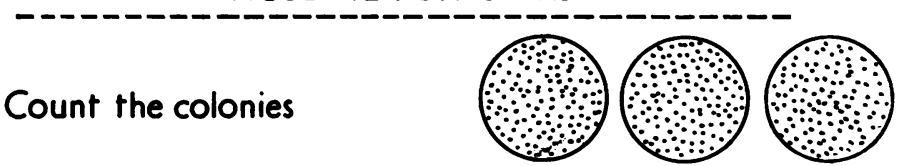

FIGURE

Experimental procedure 
addition of $5 \mathrm{ml}$. of neutralizer. After $30 \mathrm{sec}$. the final mixture was plated out in triplicate onto lysed horse blood agar using a standard platinum loop. To ensure that the neutralizer did not itself inhibit the organisms, a control of gonococci plus neutralizer was included and further Miles and Misra counts were carried out as before for comparison with those of the initial inoculum (Miles and others, 1938).

\section{Results}

Table I illustrates the percentage kill of $N$. gonorrhoeae by various dilutions of Hibitane. In this first experiment the strains used were unselected and both Types 1 and 4 were randomly distributed among the numbers sampled. As can be seen from the results, there was a reasonably high percentage kill in the cases of all strains; however, strains T5, 24 , and 26 were exceptionally sensitive to the action of Hibitane giving 100 per cent. kill dilutions as low as $1 / 5,000$, an extremely low concentration for this antiseptic. On further investigation all of these strains were found to be Type 1 and consequently a comparison of the action of Hibitane on Type 1 and 4 variants of seven strains was undertaken. The findings are shown in Table II. The dilutions of Hibitane were varied from $1 / 1,000$ to $1 / 10,000$, and the sensitivity of Types 1 and 4 compared. It is interesting to note that in almost all instances Type 1 variants were much more sensitive to the killing action of Hibitane than were Type 4 variants.

The foregoing results were obtained using suspending medium at $\mathrm{pH} 7 \cdot 3$. For Hibitane to be an effective bactericidal agent for prophylaxis, it must be equally effective at the normal lower vaginal $\mathrm{pH}$ of 4.0 to 5.0 . Table III gives the results of a further comparison of Types 1 and 4 using as diluent serum broth at $\mathrm{pH} 4 \cdot 5$. Very little impairment of the action of Hibitane was observed at the lower $\mathrm{pH}$ and Type 1 again appeared to be more sensitive than Type 4.

\section{Discussion}

Gonococci are extremely sensitive to the bactericidal action of Hibitane gluconate solution, even at low concentrations. Furthermore, virulent colonies of Type 1 appear to be much more susceptible to this action than those of the avirulent Type 4.

These morphological differences were shown to be associated with variations in virulence of the gonococcal colonies by Kellogg and his colleagues who demonstrated that colonies of Types 1 and 2 would cause a urethral discharge in male volunteers, while those of Types 3 and 4 would not (Kellogg, Peacock, Deacon, Brown, and Pirkle, 1963). Until recently, there was no known biochemical or cytological explanation for these morphological dissimilarities. However, in 1971, Swanson showed that pili could be found in greater numbers on Types 1 and 2 gonococci, whereas Types 3 and 4 possessed very few of these structures (Swanson, Kraus, and Gotschlich, 1971).

More recently, Cheng, Kwapinski, and Ronald (1974) demonstrated that Type 1 colonies contained a unique protein which was extremely toxic to chick embryos. This protein could not be found in colonies

TABLE I Percentage kill of N. gonorrhoeae at various concentrations of 20 per cent. Hibitane gluconate solution

\begin{tabular}{|c|c|c|c|c|c|c|c|}
\hline \multirow[t]{2}{*}{ Strain no. } & \multirow{2}{*}{$\begin{array}{l}\text { Initial } \\
\text { inoculum }\end{array}$} & \multicolumn{5}{|c|}{ Percentage kill by Hibitane ( final concentrations) } & \multirow{2}{*}{$\begin{array}{l}\text { Control } \\
\text { (orgs/ml. } \\
\text { recovered) }\end{array}$} \\
\hline & & $1 / 1,000$ & $1 / 2,000$ & $1 / 3,000$ & $1 / 4,000$ & $1 / 5,000$ & \\
\hline $\begin{array}{c}42 \\
\text { T6 } \\
1 \\
3 \\
55 \\
41 \\
62 \\
33\end{array}$ & $\begin{array}{r}1 \times 10^{4} \\
1 \times 10^{5} \\
15 \times 10^{3} \\
54 \times 10^{4} \\
13 \times 10^{4} \\
36 \times 10^{4} \\
9 \times 10^{3} \\
1 \times 10^{4}\end{array}$ & $\begin{array}{c}100 \\
100 \\
100 \\
99 \cdot 1 \\
99 \cdot 8 \\
98 \cdot 7 \\
100 \\
100\end{array}$ & $\begin{array}{c}99 \cdot 7 \\
99 \cdot 41 \\
99 \cdot 89 \\
96 \cdot 2 \\
99 \cdot 6 \\
97 \cdot 6 \\
100 \\
99 \cdot 8\end{array}$ & $\begin{array}{l}99 \cdot 5 \\
93 \cdot 6 \\
99 \cdot 73 \\
95 \cdot 88 \\
99 \cdot 3 \\
95 \cdot 1 \\
99 \cdot 1 \\
99 \cdot 7\end{array}$ & $\begin{array}{l}98 \cdot 2 \\
87 \cdot 2 \\
96 \cdot 4 \\
93 \cdot 2 \\
98 \cdot 9 \\
94 \cdot 2 \\
98 \cdot 0 \\
99 \cdot 6\end{array}$ & $\begin{array}{l}96 \cdot 0 \\
86 \cdot 1 \\
94 \cdot 0 \\
89 \cdot 9 \\
92 \cdot 0 \\
93 \cdot 1 \\
94 \cdot 9 \\
99 \cdot 7\end{array}$ & $\begin{array}{r}1 \times 10^{4} \\
1 \times 10^{5} \\
13 \times 10^{3} \\
62 \times 10^{4} \\
11 \times 10^{4} \\
14 \times 10^{4} \\
1 \times 10^{3} \\
95 \times 10^{4}\end{array}$ \\
\hline T5 & $39 \times 10^{5}$ & 100 & 100 & 100 & 100 & 100 & $41 \times 10^{5}$ \\
\hline $\begin{array}{l}\text { T4 } \\
\text { T3 } \\
21 \\
22 \\
23 \\
24\end{array}$ & $\begin{array}{r}101 \times 10^{4} \\
54 \times 10^{4} \\
14 \times 10^{4} \\
44 \times 10^{3} \\
42 \times 10^{3} \\
36 \times 10^{5}\end{array}$ & $\begin{array}{c}100 \\
100 \\
99 \cdot 8 \\
99 \cdot 9 \\
100 \\
100\end{array}$ & $\begin{array}{l}99.4 \\
99.8 \\
99 \cdot 5 \\
99 \cdot 6 \\
98 \cdot 5 \\
99 \cdot 8\end{array}$ & $\begin{array}{l}97 \cdot 1 \\
99.6 \\
98 \cdot 9 \\
99 \cdot 3 \\
84 \cdot 0 \\
87 \cdot 5 \\
\end{array}$ & $\begin{array}{l}96 \cdot 0 \\
98.7 \\
98 \cdot 1 \\
99 \cdot 2 \\
72 \cdot 0 \\
96 \cdot 8 \\
\end{array}$ & $\begin{array}{l}95 \cdot 3 \\
96.9 \\
97 \cdot 4 \\
99 \cdot 8 \\
50 \cdot 0 \\
95 \cdot 4 \\
\end{array}$ & $\begin{array}{l}98 \times 10^{4} \\
57 \times 10^{4} \\
13 \times 10^{4} \\
56 \times 10^{3} \\
42 \times 10^{3} \\
15 \times 10^{5} \\
\end{array}$ \\
\hline $\begin{array}{l}25 \\
26\end{array}$ & $\begin{array}{l}4 \times 10^{4} \\
6 \times 10^{4}\end{array}$ & $\begin{array}{l}100 \\
100\end{array}$ & $\begin{array}{l}100 \\
100\end{array}$ & $\begin{array}{l}100 \\
100\end{array}$ & $\begin{array}{l}100 \\
100\end{array}$ & $\begin{array}{l}100 \\
100\end{array}$ & $\begin{array}{r}9 \times 10^{4} \\
13 \times 10^{4} \\
\end{array}$ \\
\hline $\begin{array}{l}27 \\
28 \\
29 \\
30 \\
31 \\
32 \\
34 \\
35 \\
36\end{array}$ & $\begin{aligned} 54 & \times 10^{4} \\
5 & \times 10^{3} \\
9 & \times 10^{5} \\
89 & \times 10^{4} \\
4 & \times 10^{4} \\
3 & \times 10^{3} \\
21 & \times 10^{5} \\
9 & \times 10^{3} \\
21 & \times 10^{4}\end{aligned}$ & $\begin{array}{c}100 \\
100 \\
100 \\
99.9 \\
100 \\
100 \\
100 \\
100 \\
100\end{array}$ & $\begin{array}{c}100 \\
99 \cdot 81 \\
100 \\
98.3 \\
100 \\
99 \cdot 9 \\
98 \cdot 3 \\
99 \cdot 1 \\
98 \cdot 2\end{array}$ & $\begin{array}{c}99.8 \\
96 \cdot 2 \\
100 \\
96.1 \\
99 \cdot 3 \\
100 \\
89 \cdot 4 \\
99 \cdot 1 \\
96 \cdot 5\end{array}$ & $\begin{array}{c}99.2 \\
98 \cdot 7 \\
98 \cdot 1 \\
97.3 \\
99 \cdot 1 \\
99 \cdot 6 \\
73 \cdot 0 \\
96 \cdot 6 \\
95 \cdot 4\end{array}$ & $\begin{array}{c}97.9 \\
95 \cdot 1 \\
96 \cdot 5 \\
94.1 \\
97 \cdot 9 \\
99 \cdot 0 \\
51 \cdot 9 \\
95 \cdot 3 \\
93 \cdot 2\end{array}$ & $\begin{aligned} 60 & \times 10^{4} \\
1 & \times 10^{3} \\
18 & \times 10^{5} \\
1 & \times 10^{4} \\
3 & \times 10^{4} \\
4 & \times 10^{3} \\
25 & \times 10^{5} \\
3 & \times 10^{3} \\
28 & \times 10^{4}\end{aligned}$ \\
\hline
\end{tabular}


TABLE II Effect of various dilutions of 20 per cent. Hibitane gluconate solution on gonococcal Types 1 and 4

\begin{tabular}{|c|c|c|c|c|c|c|c|c|c|}
\hline \multirow{2}{*}{$\begin{array}{l}\text { Strain } \\
\text { no. }\end{array}$} & \multirow[t]{2}{*}{ Type } & \multirow{2}{*}{$\begin{array}{l}\text { Initial } \\
\text { inoculum }\end{array}$} & \multicolumn{6}{|c|}{ Percentage kill by Hibitane (concentrations) } & \multirow{2}{*}{$\begin{array}{l}\text { Control } \\
\text { (Orgs/ml. } \\
\text { recovered) }\end{array}$} \\
\hline & & & $1 / 1,000$ & $1 / 3,000$ & $1 / 5,000$ & $1 / 7,000$ & $1 / 9,000$ & $1 / 10,000$ & \\
\hline 9 & $\begin{array}{l}1 \\
4\end{array}$ & $\begin{array}{l}7 \times 10^{4} \\
4 \times 10^{4}\end{array}$ & $\begin{array}{l}100 \\
100\end{array}$ & $\begin{array}{l}100 \\
99 \cdot 1\end{array}$ & $\begin{array}{l}100 \\
98 \cdot 7\end{array}$ & $\begin{array}{l}100 \\
96.8\end{array}$ & $\begin{array}{l}99 \cdot 5 \\
95 \cdot 4\end{array}$ & $\begin{array}{l}97 \cdot 3 \\
89 \cdot 1\end{array}$ & $\begin{array}{l}7 \times 10^{4} \\
4 \times 10^{4}\end{array}$ \\
\hline 10 & $\begin{array}{l}1 \\
4\end{array}$ & $\begin{array}{l}10 \times 10^{4} \\
14 \times 10^{4}\end{array}$ & $\begin{array}{l}100 \\
100\end{array}$ & $\begin{array}{l}100 \\
99.8\end{array}$ & $\begin{array}{l}100 \\
97 \cdot 4\end{array}$ & $\begin{array}{l}100 \\
95.8\end{array}$ & $\begin{array}{l}100 \\
93 \cdot 2\end{array}$ & $\begin{array}{l}99 \cdot 8 \\
90 \cdot 1\end{array}$ & $\begin{array}{l}11 \times 10^{4} \\
16 \times 10^{4}\end{array}$ \\
\hline 12 & $\begin{array}{l}1 \\
4\end{array}$ & $\begin{array}{l}10 \times 10^{4} \\
14 \times 10^{4}\end{array}$ & $\begin{array}{l}100 \\
99 \cdot 1\end{array}$ & $\begin{array}{l}100 \\
96.4\end{array}$ & $\begin{array}{l}100 \\
98 \cdot 1\end{array}$ & $\begin{array}{l}100 \\
95 \cdot 1\end{array}$ & $\begin{array}{l}100 \\
91.8\end{array}$ & $\begin{array}{l}100 \\
86 \cdot 1\end{array}$ & $\begin{array}{r}16 \times 10^{4} \\
1 \times 10^{4}\end{array}$ \\
\hline 25 & $\begin{array}{l}1 \\
4\end{array}$ & $\begin{array}{l}6 \times 10^{4} \\
9 \times 10^{4}\end{array}$ & $\begin{array}{l}100 \\
100\end{array}$ & $\begin{array}{l}100 \\
99 \cdot 8\end{array}$ & $\begin{array}{l}100 \\
97.0\end{array}$ & $\begin{array}{l}100 \\
89 \cdot 6\end{array}$ & $\begin{array}{l}99 \cdot 1 \\
84 \cdot 2\end{array}$ & $\begin{array}{l}98 \cdot 1 \\
81.9\end{array}$ & $\begin{array}{r}13 \times 10^{4} \\
1 \times 10^{4}\end{array}$ \\
\hline 26 & $\begin{array}{l}1 \\
4\end{array}$ & $\begin{array}{l}13 \times 10^{5} \\
13 \times 10^{5}\end{array}$ & $\begin{array}{l}100 \\
100\end{array}$ & $\begin{array}{l}100 \\
99 \cdot 8\end{array}$ & $\begin{array}{l}100 \\
84 \cdot 2\end{array}$ & $\begin{array}{l}100 \\
46.0\end{array}$ & $\begin{array}{l}99 \cdot 8 \\
40 \cdot 1\end{array}$ & $\begin{array}{l}99.8 \\
36.9\end{array}$ & $\begin{array}{l}12 \times 10^{5} \\
16 \times 10^{5}\end{array}$ \\
\hline 11 & $\begin{array}{l}1 \\
4\end{array}$ & $\begin{aligned} 14 & \times 10^{4} \\
6 & \times 10^{5}\end{aligned}$ & $\begin{array}{l}100 \\
100\end{array}$ & $\begin{array}{l}100 \\
100\end{array}$ & $\begin{array}{c}99 \cdot 1 \\
100\end{array}$ & $\begin{array}{l}96 \cdot 1 \\
99 \cdot 8\end{array}$ & $\begin{array}{l}93 \cdot 4 \\
96 \cdot 7\end{array}$ & $\begin{array}{l}90 \cdot 2 \\
96 \cdot 0\end{array}$ & $\begin{array}{r}16 \times 10^{4} \\
5 \times 10^{5}\end{array}$ \\
\hline 16 & $\begin{array}{l}1 \\
4\end{array}$ & $\begin{array}{l}13 \times 10^{5} \\
15 \times 10^{4}\end{array}$ & $\begin{array}{l}100 \\
99.9\end{array}$ & $\begin{array}{l}100 \\
98 \cdot 3\end{array}$ & $\begin{array}{l}100 \\
96 \cdot 5\end{array}$ & $\begin{array}{l}100 \\
95.9\end{array}$ & $\begin{array}{l}100 \\
94 \cdot 3\end{array}$ & $\begin{array}{l}100 \\
92 \cdot 1\end{array}$ & $\begin{array}{l}14 \times 10^{5} \\
36 \times 10^{4}\end{array}$ \\
\hline
\end{tabular}

TABLE III Effect of various dilutions of 20 per cent. Hibitane gluconate solution on gonococcal Types 1 and 4 using Lab Lemco and horse serum at pH 4.5

\begin{tabular}{|c|c|c|c|c|c|c|c|c|c|}
\hline \multirow{2}{*}{$\begin{array}{l}\text { Strain } \\
\text { no. }\end{array}$} & \multirow[t]{2}{*}{ Type } & \multirow{2}{*}{$\begin{array}{l}\text { Initial } \\
\text { inoculum }\end{array}$} & \multicolumn{6}{|c|}{ Percentage kill by Hibitane (concentrations) } & \multirow{2}{*}{$\begin{array}{l}\text { Control } \\
\text { (Orgs } / m l . \\
\text { recovered) }\end{array}$} \\
\hline & & & $1 / 1,000$ & $1 / 3,000$ & $1 / 5,000$ & $1 / 7,000$ & $1 / 9,000$ & $1 / 10,000$ & \\
\hline 9 & $\begin{array}{l}1 \\
4\end{array}$ & $\begin{array}{l}15 \times 10^{4} \\
16 \times 10^{4}\end{array}$ & $\begin{array}{l}100 \\
100\end{array}$ & $\begin{array}{l}100 \\
99.8\end{array}$ & $\begin{array}{l}100 \\
97.5\end{array}$ & $\begin{array}{l}100 \\
95 \cdot 4\end{array}$ & $\begin{array}{l}97 \cdot 8 \\
95 \cdot 4\end{array}$ & $\begin{array}{l}95 \cdot 2 \\
88 \cdot 9\end{array}$ & $\begin{array}{l}12 \times 10^{4} \\
19 \times 10^{4}\end{array}$ \\
\hline 10 & $\begin{array}{l}1 \\
4\end{array}$ & $\begin{array}{l}10 \times 10^{3} \\
15 \times 10^{3}\end{array}$ & $\begin{array}{l}100 \\
100\end{array}$ & $\begin{array}{l}100 \\
100\end{array}$ & $\begin{array}{l}100 \\
99 \cdot 2\end{array}$ & $\begin{array}{l}99 \cdot 5 \\
96 \cdot 3\end{array}$ & $\begin{array}{l}95 \cdot 9 \\
94 \cdot 8\end{array}$ & $\begin{array}{l}95 \cdot 1 \\
90 \cdot 1\end{array}$ & $\begin{array}{l}13 \times 10^{3} \\
15 \times 10^{3}\end{array}$ \\
\hline 12 & $\begin{array}{l}1 \\
4\end{array}$ & $\begin{array}{l}1 \times 10^{3} \\
3 \times 10^{3}\end{array}$ & $\begin{array}{l}100 \\
100\end{array}$ & $\begin{array}{l}100 \\
99 \cdot 8\end{array}$ & $\begin{array}{l}100 \\
97.0\end{array}$ & $\begin{array}{l}100 \\
89 \cdot 5\end{array}$ & $\begin{array}{l}100 \\
84 \cdot 8\end{array}$ & $\begin{array}{l}100 \\
83.0\end{array}$ & $\begin{array}{l}4 \times 10^{3} \\
1 \times 10^{3}\end{array}$ \\
\hline 25 & $\begin{array}{l}1 \\
4\end{array}$ & $\begin{array}{l}6 \times 10^{5} \\
9 \times 10^{4}\end{array}$ & $\begin{array}{l}100 \\
99.8\end{array}$ & $\begin{array}{l}100 \\
96 \cdot 2\end{array}$ & $\begin{array}{l}100 \\
80 \cdot 2\end{array}$ & $\begin{array}{l}99 \cdot 4 \\
53 \cdot 0\end{array}$ & $\begin{array}{l}98 \cdot 9 \\
45 \cdot 1\end{array}$ & $\begin{array}{l}97 \cdot 0 \\
36 \cdot 8\end{array}$ & $\begin{array}{r}1 \times 10^{5} \\
1 \times 10^{4}\end{array}$ \\
\hline 26 & $\begin{array}{l}1 \\
4\end{array}$ & $\begin{array}{l}4 \times 10^{4} \\
5 \times 10^{4}\end{array}$ & $\begin{array}{l}100 \\
100\end{array}$ & $\begin{array}{l}100 \\
99 \cdot 8\end{array}$ & $\begin{array}{l}100 \\
90.0\end{array}$ & $\begin{array}{l}99 \cdot 4 \\
89 \cdot 3\end{array}$ & $\begin{array}{l}98 \cdot 6 \\
86 \cdot 1\end{array}$ & $\begin{array}{l}98 \cdot 2 \\
85 \cdot 0\end{array}$ & $\begin{array}{l}8 \times 10^{4} \\
1 \times 10^{4}\end{array}$ \\
\hline 11 & $\begin{array}{l}1 \\
4\end{array}$ & $\begin{array}{l}11 \times 10^{4} \\
12 \times 10^{4}\end{array}$ & $\begin{array}{l}100 \\
100\end{array}$ & $\begin{array}{c}100 \\
99.9\end{array}$ & $\begin{array}{l}100 \\
99 \cdot 4\end{array}$ & $\begin{array}{l}100 \\
98.6\end{array}$ & $\begin{array}{l}96 \cdot 4 \\
97 \cdot 1\end{array}$ & $\begin{array}{l}90 \cdot 9 \\
95 \cdot 0\end{array}$ & $\begin{array}{l}9 \times 10^{4} \\
9 \times 10^{4}\end{array}$ \\
\hline$\overline{16}$ & $\begin{array}{l}1 \\
4\end{array}$ & $\begin{array}{l}13 \times 10^{3} \\
15 \times 10^{3}\end{array}$ & $\begin{array}{l}100 \\
99.9\end{array}$ & $\begin{array}{c}100 \\
98 \cdot 4\end{array}$ & $\begin{array}{l}100 \\
96 \cdot 3\end{array}$ & $\begin{array}{l}100 \\
96.0\end{array}$ & $\begin{array}{l}100 \\
94 \cdot 3\end{array}$ & $\begin{array}{l}98 \cdot 1 \\
90 \cdot 0\end{array}$ & $\begin{array}{l}14 \times 10^{3} \\
36 \times 10^{3}\end{array}$ \\
\hline
\end{tabular}

of Type 4 organisms. Waitkins (1974) showed that Types 1 and 2 would haemagglutinate human red blood cells, while Type 4 would not. The author concluded that the variation in haemagglutination power was due to the absence of any pili on the avirulent Type 4. To date these are the only distinguishing factors, other than virulence, between Kellogg's different morphological types.

Our findings indicate that Hibitane gluconate solution has a differential action on Type 1 and Type 4 gonococci. Table II illustrates the varying bactericidal effect that Hibitane gluconate has on both Types 1 and 4 of the same strains of gonococci. Although results from testing only seven strains are reported, we found the same differential effect between Types 1 and 4 in over fifty other strains tested. Furthermore, the killing effect of Hibitane was not greatly altered when Lab Lemco plus horse serum was used as diluent. These results would suggest that, at least quantitatively, there is a detectable difference in susceptibility between Kellogg's Types 1 and 4 using suitable test dilutions of Hibitane gluconate solution.

These findings suggest that Hibitane might be used as a chemical prophylactic agent in the control of gonorrhoea; however, in vivo verification of its effectiveness would be required and like all topical agents there remains the problem of ensuring that it reaches all organisms in adequate concentration.

\section{Summary}

Virulent Type 1 gonococci have been shown to be much more susceptible to the killing action of 
Hibitane gluconate than the avirulent Type 4. Those exploring the possibility of genital prophylaxis as a means of controlling gonorrhoea might find Hibitane worthy of consideration.

\section{References}

BARR, W. (1964) Brit. med. F., 1, 1361

Calman, R. M., and MurRaY, J. (1956) Ibid., 2, 200

Cheng, A., Kwapinski, J. B., and Ronald, A. R. (1974) Canad. F. Microbiol., 20, 1163

CowAN, M. E., and CREE, G. E. (1973) Brit. F. vener. Dis., 49, 65

Dulbecco, R., and Vogt, M. (1954) f. exp. Med., 99, 167

Flynn, J., and Waitkins, S. A. (1972).7. clin. Path., 25, 525

FowLER, A. W. (1963) Lancet, 1, 387

GRANT, J. C. (1968) Brit. med. f., 4, 646
I.C.I. Pharmaceuticals Division (1973) 'Hibitane (Chlorhexidine). Review of Biochemistry and Toxicology'. I.C.I., Macclesfield

Kellogg, D. S., Peacock, W. L., Deacon, W., Brown, L., and Pirkie, C. I. (1963) F. Bact., 85, 1274

LEE, T.-Y. (1974) Brit. F. vener. Dis., 50, 82

—, Utidjian, H. M. D., Singh, B., Carpenter, U., and CuTler, J. C. (1972) Ibid., 48, 376

LOWBURY, E. J. L., LiLly, H. A., and BuLL, J. P. (1960) Brit. med. F., 2, 1039

MILES, A. A., MISRA, S. S., and IRWIN, J. O. (1938) f. Hyg. (Camb.), 38, 732

MONTGOMeRY, P. R. (1964) Prescrib. F., 4, 17

Morris, G. M., and McLAREN, D. M. (1969) Brit. F. clin. Pract., 23, 349

Murray, J., and Calman, R. M. (1955) Brit. med. f., 1,81

Swanson, J., KraUs, S. J., and Gotschlich, E. (1971) F. $\exp$. Med., 134, 886

WAITKINS, S. A. (1974) Brit. f. vener. Dis., 50, 272

White, L. A., and KellogG, D. S., Jr. (1965) Appl. Microbiol., 13, 171 